\title{
Merkel cell carcinoma - pro and cons aspects for sentinel lymph node biopsy
}

\author{
Nicolae Bacalbasa ${ }^{1,2,3}$, Irina Balescu4, Mihai Dimitriu',5, Mihaela Vilcu ${ }^{2,3}$, \\ Iulian Brezean ${ }^{2,3}$ \\ ${ }^{1}$ Center of Excellence in Translational Medicine, Fundeni Clinical Institute, Bucharest, Romania \\ 2"Carol Davila" University of Medicine and Pharmacy, Bucharest, Romania \\ 3"Ion Cantacuzino" Clinical Hospital, Bucharest, Romania \\ ${ }^{4}$ Ponderas Academic Hospital, Bucharest, Romania \\ ${ }^{5}$ Pantelimon Clinical Hospital, Bucharest, Romania
}

\begin{abstract}
Merkel cell carcinoma represents an aggressive histopathological subtype of skin cancer with a high propensity to spread via lymphatic route. Once the benefits of the sentinel lymph node detection have been proven in malignant melanoma, the effectiveness of the method was investigated further on in Merkel cell carcinoma, as long as the two tumoral subtypes seem to have a common embryological origin. Surprisingly, the results reported so far in Merkel cell carcinoma are rather confusing, the method not being so far associated as part of the standard therapeutic protocol. However, it seems that the method plays a certain role in identifying the high risk patients and further tailoring the therapeutic strategy. This is a literature review of the most eloquent studies conducted on this theme.
\end{abstract}

Keywords: Merkel cell carcinoma, sentinel node biopsy, prognostic

\section{INTRODUCTION}

Merkel cell carcinoma represents a neuroendocrine neoplasm with an aggressive biological behavior and a high capacity to spread, leading in this way to the apparition of both loco-regional and distant metastases; moreover, this histopathological subtype of skin cancer is considered to be the most aggressive cutaneous malignancy (1). Moreover, it has been stated that tumoral cell spread follows an orderly progression from the initial site to the lymphatic stations and only late during disease progression to distant sites (2).

Similarly to malignant melanomas, Merkel cell carcinomas originate from neural crest cells and trend to spread via lymphatic route, the development of nodal metastases being a powerful predictor of the long-term survival (2). Due to this aggressive biology, Merkel cell carcinoma is associated with poor long term survival rates, the overall 5-year survival rate ranging between 30 and $64 \%(3-5)$.
One of the largest studies which demonstrated the influence of nodal metastases in Merkel cell carcinoma patients was published by Morrison et al. in 1990 (6). The study was conducted between 1966 and 1987 and included 54 patients with localized Merkel cell carcinoma, the five year overall survival rate being of $30 \%$; the impact of the node metastases was demonstrated by the significantly different course of patients presenting lymph node metastases when compared to the one reported in cases with histologically negative nodes; the median survival rate of the first category was of 13 months, while for the second category the median survival rate reached 40 months $(p<0,04)$. Moreover, the authors underlined the fact that the prognostic of these patients significantly improved after modifying the therapeutic protocol and routinely associating adjuvant radiation therapy of the lymphatic basins (while initially it had been proposed that only patients presenting large initial tumors or nodal involvement should undergo adjuvant radia- 
tion therapy) (6). However, the estimation of the lymph node status at that moment was done only by clinical examination.

\section{Rationale for sentinel lymph node detection in Merkel cell carcinoma}

Similarly to other solid tumors, sentinel lymph node detection in Merkel cell carcinoma intends to provide the analysis of the first lymph nodes in which the tumor drain; according to this status, the further treatment - follow-up, radiation therapy or extended lymph node dissection is tailored. In this way patients who could benefit most from an aggressive therapeutic strategy should be successfully identified (7). A randomized control trial which was published by the French authors in 2012 demonstrated the benefits of radiotherapy in Merkel cell carcinoma patients with clinically negative nodes submitted to adjuvant radiation therapy reported a significantly lower rate of regional recurrences when compared to patients submitted to close follow-up (8). Beginning from this hypothesis, the sentinel node biopsy has been proposed in order to provide a better identification of the patients who might present occult disease and who could benefit most from the association of adjuvant radiation therapy. However, data regarding the effectiveness of sentinel lymph node biopsy in Merkel cell carcinoma is scarcer due to the rarity of this histopathological subtype (9).

\section{Studies investigating the effectiveness of sentinel lymph node dissection in Merkel cell carcinoma}

An interesting study which came to demonstrate the effectiveness of sentinel lymph node biopsy in Merkel cell carcinoma patients originates from Ortin-Perez and co. and was published in 2007 in the European Journal of Surgical Oncology (10). The study included eight patients diagnosed with Merkel cell carcinoma in two hospitals and submitted to sentinel node detection and biopsy. Further on, the patients were supervised for a median period of 4.6 years (range 8 months to 10 years). In all cases intradermal radiocolloid injection in four points at the level of the scar of the primary tumor was performed one day before surgery, while short time before surgery blue dye was injected intradermally in six of the eight cases (in the other two cases blue dye was not administered due to the surgeon's choice). In all cases in which the positivity of the sentinel node was proven, the surgical procedure was completed by performing a regional extended lymph node dissection. Lymphoscintigra- phy identified at least one sentinel nodes in all cases, while in one case three sentinel nodes were discovered. Finally, all 10 sentinel nodes were successfully identified and retrieved, six of them (corresponding for the six cases in which patent blue had been injected) being also colored in blue; subsequently, the histopathological studies demonstrated the presence of nodal metastases in three cases which were further submitted to regional lymph node dissection. However, the histopathological studies demonstrated the absence of other lymph node metastases in all cases; after a median follow-up period of 4.6 years, none of the patients developed recurrent disease. For the three cases diagnosed with positive nodes, the median follow-up period was of 5.6 years, none of these cases reporting any recurrent disease. The authors demonstrated in this way the feasibility of the technique but underlined the fact that the prognostic significance of positive sentinel nodes remains unclear (none of the eight cases irrespective of the status of the sentinel node reporting metastatic disease even in the absence of radiation therapy) (10).

A more recent study which was conducted on this theme comes from Pollock and co. and was published in 2017; this time the study was focused on studying the importance of sentinel lymph node detection in high risk Merkel cell carcinoma patients (11). According to the same study group, the high risk lesions are characterized by a tumoral diameter larger than $2 \mathrm{~cm}$, recurrent disease, high grade histological features, the association with peritumoral/perineural invasion and/or the presence of a larger than $4 \mathrm{~mm}$ in depth lesion. The authors review data originating from 1618 consecutive skin cancer patients and identified 30 cases which met the above presented criteria. In all cases the dual method consisting of radiocolloid injection one day before surgery and isosulfan blue injection at the time of surgery was performed. Cases with positive nodal disease were further submitted to extended lymph node dissection, while cases with negative sentinel nodes were further evaluated for the administration of adjuvant chemotherapy or adjuvant irradiation. After performing the dual method, a median number of two sentinel nodes were identified, the median number being higher in cases with head or neck Merkel carcinomas. Among the 30 cases included in the study, there were four cases diagnosed with positive sentinel lymph nodes, two patients presenting initially with non-mucosal lip tumors and two other cases being diagnosed with forearm tumors. According to the method of the study, all four cases diagnosed with sentinel 
node metastases were further submitted to extended lymph node dissection, an average of five additional nodes being harvested; among the non-sentinel nodes there were two positive nodes (in two patients, one in each case). Cases diagnosed with positive nodes were further submitted to adjuvant radiation therapy and platinum based chemotherapy, none of them developing recurrent disease after a median follow-up period of 56 months. The univariate analysis demonstrated that the presence of positive sentinel nodes was significantly correlated with a tumoral diameter larger than $2 \mathrm{~cm}$ and a depth of invasion higher than $4 \mathrm{~mm}$, while at multivariate analysis only the depth of invasion was significantly associated with the positivity of the sentinel node status. Among the 30 cases there were five cases which developed recurrent disease, none of them presenting positive sentinel nodes. Therefore, the authors concluded that sentinel lymph node biopsy should become part of the surgical protocol in high risk patients (defined by a tumoral invasion higher than $4 \mathrm{~mm}$ and a diameter higher than $2 \mathrm{~cm}$ ). Moreover, the authors pointed out the fact that after performing the sentinel lymph node biopsy in these patients, an upgrade of the stage of the disease was realized in $13 \%$ of cases, leading to the association of the adjuvant treatment (11).

\section{Merkel cell carcinoma of the head and neck - a particular situation?}

When it comes to the role of sentinel lymph node detection in head and neck Merkel cell carcinoma, the preliminary results are conflicting. In a recent paper published by Ricard et al. in which the authors included 12 patients with head and neck lesions submitted to sentinel lymph node biopsy, a single patient proved to have a positive sentinel node; however, two cases with negative sentinel nodes experienced a regional lymphatic recurrence within the first two years, one of them being dead at the end of the study. This fact was explained by the difficulty of sampling the facial region (12). Moreover, the presence of multiple and various drainage channels at the level of the facial region was also incriminated $(7,12,13)$.

A similar conclusion was also drawn by Maher's systematic review which included 23 studies and 81 patients with head and neck Merkel cell carcinoma. After a median follow-up period of 32.8 months, $12.3 \%$ of cases with negative sentinel node biopsy developed nodal recurrences and 5\% of them developed distant recurrent disease. However, among patients with negative sentinel nodes who received adjuvant radiation therapy there was no case of recurrent disease (7).

\section{The role of indocyanine green in sentinel node detection}

In order to maximize the detection rate of the sentinel nodes in Merkel cell carcinoma, indocyanine green injection has been proposed with promising results. One of the first studies which analyzed the efficacy of indocyanine green injection was conducted in Cleveland, Ohio and included 24 consecutive patients in whom radiocolloid and indocyanine green were administrated. Their results were compared to those reported in a similar group of 11 patients submitted to radiocolloid and blue dye injection. The authors demonstrated the fact that the detection rate of the sentinel node was significantly improved in cases in which indocyanine green was associated (94.8\% versus $63.6 \%$ ); moreover, the authors underlined the fact that the indocyanine green method was able to identify certain sentinel lymph nodes which were skipped after radiocolloid injection, and concluded that this tracer might increase the accuracy of the method (14).

\section{CONCLUSIONS}

Merkel cell carcinoma represents an aggressive histopathological subtype of skin cancer, associated with high rates of relapse and poor overall survival rates. In such cases association of sentinel lymph node biopsy might provide a better identification of patients at risk to develop systemic recurrence. It seems that most often the positivity of the sentinel node is encountered in larger than $2 \mathrm{~cm}$ and deeper than $4 \mathrm{~mm}$ lesions. As for the cases diagnosed with head and neck lesions, the situation is even more confusing, no correlation between the sentinel node status and the recurrence rate being established so far; these results are mainly explained by the anatomical particularities of the lymphatic channels at the level of the cephalic region. However, further larger, prospective studies are still needed before introducing the method as part of the standard therapeutic protocol. 


\section{REFERENCES}

1. Toker C. Trabecular carcinoma of the skin. Arch.Dermatol. 1972; 105:107-10.

2. Poulsen M. Merkel-cell carcinoma of the skin. Lancet Oncol. 2004; 5:593-99.

3. Allen PJ, Bowne WB, Jaques DP et al. Merkel cell carcinoma: prognosis and treatment of patients from a single institution. J.Clin. Oncol. 2005; 23:2300-9.

4. Bichakjian CK, Lowe L, Lao CD et al. Merkel cell carcinoma: critical review with guidelines for multidisciplinary management. Cancer 2007; 110:1-12.

5. Smith DE, Bielamowicz $S$, Kagan AR et al. Cutaneous neuroendocrine (Merkel cell) carcinoma. A report of 35 cases. Am.J.Clin. Oncol. 1995; 18:199-203.

6. Morrison WH, Peters LJ, Silva EG et al. The essential role of radiation therapy in securing locoregional control of Merkel cell carcinoma. Int.J.Radiat.Oncol.Biol.Phys. 1990;19:583-91.

7. Maher NG, Murrell DF. A systematic review of patients with Merkel cell carcinoma of the head and neck and a negative sentinel lymph node biopsy. Int.J.Womens Dermatol. 2015;1:41-6.

8. Jouary T, Leyral C, Dreno B et al. Adjuvant prophylactic regional radiotherapy versus observation in stage I Merkel cell carcinoma: a multicentric prospective randomized study. Ann. Oncol. 2012; 23:1074-80.
9. Gipponi M, Solari N, Di Somma FC, Bertoglio S, Cafiero F. New fields of application of the sentinel lymph node biopsy in the pathologic staging of solid neoplasms: review of literature and surgical perspectives. J.Surg. Oncol. 2004; 85:171-9.

10. Ortin-Perez J, van Rijk MC, Valdes-Olmos RA et al. Lymphatic mapping and sentinel node biopsy in Merkel's cell carcinoma. Eur.J.Surg. Oncol. 2007; 33:119-22.

11. Pollock J, Pollock AE, Hardin R et al. Sentinel node lymphoscintigraphy in high-risk cutaneous squamous cell carcinoma. Clinical Skin Cancer. 2017; 1(2): 88-93.

12. Ricard AS, Sessiecq $Q$, Siberchicot $F$ et al. Sentinel lymph node biopsy for head and neck Merkel cell carcinoma: a preliminary study. Eur.Ann.Otorhinolaryngol.Head Neck Dis. 2015; 132:77-80.

13. O'Brien CJ, Uren RF, Thompson JF et al. Prediction of potential metastatic sites in cutaneous head and neck melanoma using lymphoscintigraphy. Am.J.Surg. 1995; 170:461-6.

14. Knackstedt RW, Knackstedt T, Gastman B. Utilization of Indocyanine Green to Aid in Identifying Sentinel Lymph Nodes in Merkel Cell Cancer. J.Surg.Res. 2018;232:365-8. 